\title{
Consensus Paper-ICIS Expert Meeting Basel 2009 treatment milestones in immune thrombocytopenia
}

\author{
Hannah Tamary • Jelena Roganovic • Meera Chitlur • \\ Diane J. Nugent
}

Received: 10 December 2009 / Accepted: 4 March 2010/Published online: 1 April 2010

(C) The Author(s) 2010. This article is published with open access at Springerlink.com

\begin{abstract}
The rarity of severe complications of this disease in children makes randomized clinical trials in immune thrombocytopenia (ITP) unfeasible. Therefore, the current management recommendations for ITP are largely dependent on clinical expertise and observations. As part of its discussions during the Intercontinental Cooperative ITP Study Group Expert Meeting in Basel, the Management working group recommended that the decision to treat an ITP patient be individualized and based mainly on bleeding symptoms and not on the actual platelet count number and should be supported by bleeding scores using a validated assessment tool. The group stressed the need to develop a uniform validated bleeding score system and to explore new measures to evaluate bleeding risk in thrombocytopenic patients - the role of rituximab as a splenectomy-sparing agent in resistant disease was also discussed. Given the apparently high recurrence rate to rituximab therapy in
\end{abstract}

\section{H. Tamary}

Pediatric Hematology Oncology Center, Schneider Children's

Medical Center of Israel, Petach Tikva and Sackler

Faculty of Medicine, Tel Aviv University,

Tel Aviv, Israel

\section{J. Roganovic}

Division of Hematology and Oncology,

University Children's Hospital,

Rijeka, Croatia

\section{Chitlur}

Department of Pediatric Hematology/Oncology,

Children's Hospital of Michigan Detroit,

Detroit, MI, USA

\section{J. Nugent $(\bowtie)$}

Pediatric Hematology,

CHOC Children's Hospital and University of California,

Irvine, CA, USA

e-mail: tomdiane@cox.net children and the drug's possible toxicity, the group felt that until more data are available, a conservative approach may be considered, reserving rituximab for patients who failed splenectomy. More studies of the effectiveness and side effects of drugs to treat refractory patients, such as TPO mimetics, cyclosporine, mycophenolate mofetil, and cytotoxic agents are required, as are long-term data on postsplenectomy complications. In the patient with either acute or chronic ITP, using a more personalized approach to treatment based on bleeding symptoms rather than platelet count should result in less toxicity and empower both physicians and families to focus on quality-of-life.

Keywords Immune $\cdot$ Thrombocytopenia $\cdot$ Treatment . Outcomes

\section{Introduction}

Immune-mediated thrombocytopenia (ITP) is an acquired disease of children and adults characterized by a transient or persistent decrease in platelet count and increased risk of bleeding. The rarity of severe complications of the disease in children makes randomized clinical prevention trials unfeasible [1]. Therefore, at present, definitions of disease severity and recommendations for management are largely dependent on clinical expertise and observations and vary considerably between countries [1, 2].

The Management Working group at the 2009 Intercontinental Cooperative ITP Study Group (ICIS) Expert Meeting in Basel discussed management milestones in ITP therapy. The group suggested that the decision to treat a patient with ITP be individualized and based mainly on bleeding symptoms and guided by patient quality-of-life. The role of rituximab as a splenectomy-sparing agent in refractory patients was 
reviewed in respect to efficacy and toxicity as were other less commonly used regimens such as chemotherapy or TPO mimetics. The best treatment for patients with chronic, resistant disease is still unclear, partly because of a lack of clinical and long-term follow-up data.

\section{First milestone}

Observation or intervention in severely thrombocytopenic patients

Decisions regarding the management of ITP have traditionally been made on the basis of the platelet count. Patients typically present with severe thrombocytopenia at diagnosis (platelet count $<20,000 / \mathrm{mm}^{3}$ ), and physicians, associating the low platelet count with a high risk of hemorrhage, often administer drug therapy. The ICIS Registry data suggest, however, that the incidence of intracranial hemorrhage, the most severe complication of childhood ITP and the primary cause of death, is very low, in the range of $0.17 \%$ of affected children [3]. A similarly low figure of $0.2 \%$ was reported by Lilleyman [4]. Bolton-Maggs and Moon [1, 4] found that the majority of bleeding symptoms in ITP are relatively minor in nature. Additionally, studies based on the ICIS prospective registry indicated that even with profound thrombocytopenia (platelet count $\leq 20,000 / \mathrm{mm}^{3}$ ), severe bleeding is uncommon at diagnosis $(2.9 \%)$, and it occurs only rarely during the next 4 weeks $(0.6 \%)$, irrespective of the treatment given [5].

Given there is minimal-to-no hemorrhage in most patients with ITP, specific descriptive [1], or semiquantitative [6,7] bleeding scoring systems have been developed. Using a descriptive approach, Bolton-Maggs and Moon [4] divided bleeding signs in newly diagnosed patients into four categories: none, mild, moderate, and severe [1]. Buchanan and Adix [5] formulated a bleeding score that assessed four domains: overall bleeding tendency, bleeding from the oral cavity, epistaxis, and cutaneous hemorrhage. Each site was graded from 0 (no bleeding) to 4 (severe bleeding). Both these instruments yielded a $3 \%$ rate of severe hemorrhage in patients with ITP $[4,5]$. However, neither has been validated in a large prospective study.

\section{Working group discussion}

The working group recommended that the decision to treat a patient with ITP should not be based on number of platelets; rather, it should be individualized and based mainly on bleeding symptoms, supported by bleeding scores (Fig. 1). In the absence of hemorrhage, i.e., a bleeding score of $0-3$ [6] or a rating of mild to moderate [1], close observation alone may be sufficient. The rare danger of severe bleeding and the side effects of treatment should be discussed at length with the family, and the parents should be educated to look for signs of bleeding and to rapidly return to the hospital should they occur.

There are extenuating circumstances that might prompt the physician to treat despite the absence of significant bleeding symptoms. If the family is geographically isolated or unable to closely observe the child, the clinician should consider treatment to increase the platelet count above $20,000 / \mathrm{mm}^{3}$ and shorten the duration of thrombocytopenia. Age should also be taken into account: an active toddler who constantly bumps into things may need therapy whereas an older child with the same bleeding pattern may not. Another major factor is quality-of-life; for example, a young athlete may need therapy to prevent bleeding into vital organs. In the
Fig. 1 Individualized decision making in ITP therapy. Circumstances in which decision to treat patients despite the absence of active bleeding are highlighted

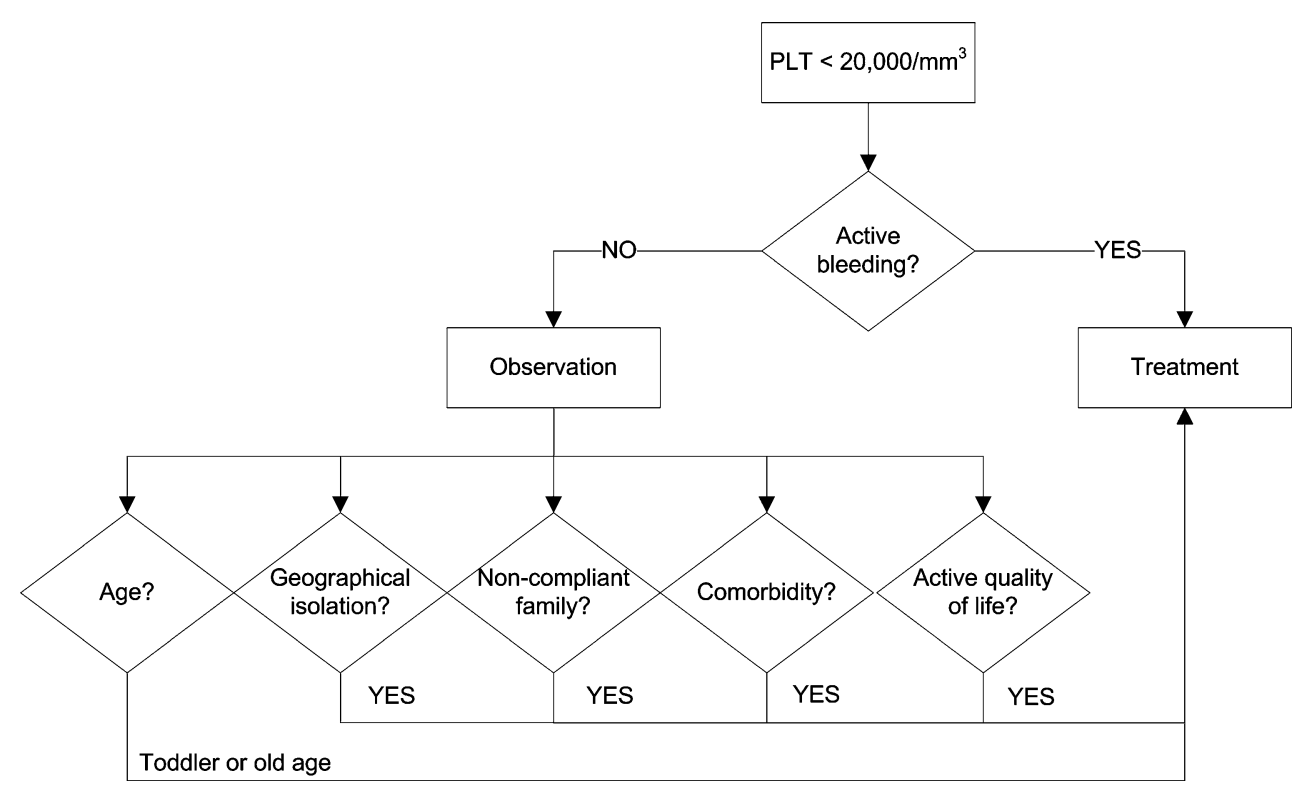


adult or child, the presence of other co-morbidities, especially those necessitating antiplatelet drugs (coronary stent, cerebrovascular disease), may be an indication to initiate therapy. Whether observing or treating, measures should be taken to correct iron deficiency anemia in order to elevate hemoglobin and improve viscosity. Conversely, the use of anti-fibrinolytic therapy should be advocated to avoid additional risks of bleeding in conjunction with the decreased platelet count.

Even if the clinician decided not to commit the patient to long-term therapy, many felt that it would be reasonable to find a treatment to which the patient is responsive initially, so that it can be applied promptly in case of an urgent need to increase platelet counts (e.g., traumatic episode or surgery).

The working group stressed the need for a uniform validated bleeding score system in ITP. Until one is developed, the group suggested that current bleeding scores be used consistently in the initial and ongoing assessments of each patient. The results will contribute to the decision to initiate therapy and are a prerequisite for prospective and retrospective data evaluation. Methods for evaluation of platelet functions in thrombocytopenic patients using global clotting measures such as thrombin generation, rotational thromboelastography, and microparticles enumeration should be explored to find an additional means to assess overall bleeding risk.

\section{Second milestone}

Therapy and its side effects

When ITP therapy is required, the first-line drugs commonly include steroids, intravenous immunoglobulin, or anti-D immunoglobulin. The working group suggested that if steroids are used, only a short course should be administered in order to avoid the well-known adverse effects of protracted corticosteroid therapy.

Treatment of ITP is indicated in only the minority of patients who have low platelet counts associated with bleeding symptoms. First-line ITP medications have been administered to patients with chronic disease on a monthly basis. In a small group of patients, second-line drugs (e.g., rituximab) or splenectomy may be considered. The decision should be individualized for the patient and his family, taking into account the high rate of spontaneous remission in childhood ITP [8], quality-of-life parameters, and treatment side effects. Splenectomy is successful in approximately $75 \%$ of pediatric patients [9]. However, the use of surgery has declined due to increased awareness of the natural history of the disease, the inability to predict a positive outcome following surgery, and the irreversible nature of the procedure coupled to the slight increased risk of sepsis [10].
In patients who fail splenectomy, cyclosporine [11], mycophenolate mofetil [12], 6-mercaptopurine [13], vincristine, and cyclophosphamide [14] have all been tried, with variable results. Prospective studies are difficult to conduct given the relative rarity of severe chronic ITP, its multiple treatment approaches, and the spontaneous remissions that may occur without medical therapy.

The sections that follow summarize working group discussions surrounding some controversial issues in chronic ITP therapy, including the role of rituximab and its potential to spare patients splenectomy. They highlighted the need for more data on the effectiveness and side effects of drugs such as cyclophosphamide, mycophenolate mofetil, and cytotoxic agents as well as long-term data on post-splenectomy complications including the possible occurrence of thrombotic complications [15].

\section{Rituximab therapy in ITP}

Rituximab is a chimeric human-mouse monoclonal antibody directed against the CD20 antigen on the surface of $\mathrm{B}$ lymphocytes. Because it induces B-cell destruction, it is assumed to stop the production of autoantibodies and platelet destruction [16]. The drug is usually well tolerated with minimal side effects. However, although the response rate to treatment is initially relatively high (40-60\%), only $15-20 \%$ of adult patients sustain a good response for at least 3 years [17]. In children, only a few studies have examined the long-term results of rituximab, and the response rate ranged from $30 \%$ to $60 \%[18,19]$.

The number of repeated courses of rituximab that may be given, their efficacy, and side effects are not well known. Furthermore, the effectiveness of a lower-than-standard dose $\left(375 \mathrm{mg} / \mathrm{m}^{2}\right.$ every 7 days for 4 weeks) remains unclear. One study reported that a lower dose of rituximab, $100 \mathrm{mg}$ IV weekly for 4 weeks, had similar activity to the standard dose [20].

Further research is needed to determine if rituximab can effectively and safely prevent the need for splenectomy. Only one study of 60 adult patients yielded promising results [21].

In February 2006, 9 years after the drug received its initial Food and Drug Administration approval, the labeling for rituximab was changed, because patients with non-Hodgkin lymphoma had developed serious viral infections after treatment with the drug. The infections included hepatitis B, cytomegalovirus, herpes simplex virus, varicella zoster virus, West Nile virus, and JC virus [22]. A recent study described the development of progressive multifocal leukoencephalopathy, a rare demyelinating disease of the central nervous system caused by reactivation of latent JC polyoma virus, following rituximab treatment, in one patient with ITP and in 57 patients with mostly lymphoid malignancies [23]. 
Working group discussion

Given the general impression that the response rate to rituximab is disappointingly low in children with ITP, together with concerns regarding the drug's toxicity and reports of progressive leukoencephalopathy, the working group suggested that until more data become available, a more conservative approach may be considered, reserving rituximab for patients who fail splenectomy. More data are needed to guide physicians in the optimal dose and whether it requires division into four weekly doses as well.

Reimmunization and antibiotic prophylaxis following splenectomy

Splenectomy poses an increased risk of septic complications which are associated with a high mortality rate. The most serious complication is overwhelming postsplenectomy infection, although its frequency has dropped due to aggressive pneumococcal vaccination, prophylactic penicillin, and prompt administration of parenteral antibiotics when fever occurs [24-26].

\section{Working group discussion}

At present, the immunization schedule after splenectomy is based on expert opinion and is not evidence-based. In the USA, experts recommend that immunization with the 23valent pneumococcal vaccine be repeated once, 5 years after the initial dose. However, this practice, as well as the duration of oral penicillin prophylaxis, varies among countries. There is a need for long-term follow-up studies of splenectomized patients and for uniform recommendations which can be prospectively evaluated.

Thrombopoietin-mimetic therapy

The newly developed second-generation thrombopoietinreceptor agonists, eltrombopag and romiplostim, have been shown to safely increase and maintain platelet production in adult patients with refractory ITP [27]. However, the longterm side-effects are still being assessed, and the exact role of these agents in the overall treatment strategy of chronic ITP remains to be established. After discontinuation of therapy, the platelet count returns to baseline in the majority of patients. Optimal weaning schedule has not been studied, and there is no prospective means to identify which patients might sustain their own platelet count without these medications. Studies to evaluate the use of TPO mimetics in combination with immune modulation may improve long-term outcome of all medications.

As the TPO-mimetic drugs are still not approved for use in children, most participants in the working group had had no experience with them. Questions regarding their application were, therefore, not discussed at length.

\section{Third milestone}

Health-related quality-of-life

Physicians caring for patients with ITP tend to focus on the platelet count rather than bleeding symptoms and may fail to appreciate the adverse effect of the treatment side effects on patient and family quality-of-life. Studies in the USA have shown that the health-related quality-of-life (HRQOL) of adults with ITP was significantly worse than that of the general population and scored similar to findings in patients with chronic diseases, such as arthritis and diabetes [28, 29].

A modified HRQOL tool for use in children (Kid's ITP Tool) was recently developed and validated [30]. The results suggested that quality-of-life of children with ITP is not significantly affected by the platelet count. Thus, it is apparently not the number of platelets per se that exerts the overall disease impact on patients and their family, but rather other factors, such as the restrictions in activity, medication side effects, multiple clinic visits, and venipunctures for laboratory studies. Applying both bleeding severity and HRQOL tools would be beneficial in guiding clinical decisions [31] and improve overall quality-of-life for these patients.

Prompted by these findings, the working group suggested that the assessment of therapeutic effectiveness should take patient and family quality-of-life into account. Indeed, the success of ITP therapy should be redefined as the ability of the patient to live life to the fullest, with minimal impact from the treatment or the disease. To achieve this goal, more quality-of-life data need to be gathered in this important field with collaborative studies incorporating the validated bleeding score and treatment regimens versus observation alone.

\section{Conclusions and future directions}

The working group recommends that the decision to treat a patient with ITP be based on the presence of active bleeding and not on the platelet count. Bleeding tendency should be further assessed by user-friendly validated bleeding scores, which are currently not widely used. The decision to treat or not to treat a severely thrombocytopenic patient needs to be family based and individualized, taking specific risk factors such as geographic isolation, age, comorbidities, and quality-of-life into consideration. In addition, more time should be spent with the family to 
carefully educate and explain the risks of observation alone versus therapy, drug side effects, and toxicities.

The decision to use specific second-line therapy should also be individualized. The effectiveness of therapy should be assessed regularly with ITP-QOL studies. Future development of specific methods to assess the risk of bleeding in thrombocytopenic patients using global clotting assays will further improve patient management.

In view of the recent report of neurological complications induced by rituximab, multiple cycles of this drug should probably not be given prior to splenectomy, which by contrast is relatively safe with immunizations and has a long-term resolution of thrombocytopenia in $75 \%$ of patients. More clinical studies are indicated to define the role of rituximab therapy in chronic ITP. In light of the varied approach to prophylaxis, it is anticipated that longterm follow-up data through ICIS will help to fully evaluate splenectomy complications. Future use of TPO-mimetic agents in children, although not curative alone, may be beneficial in refractory cases.

Overall, the working group credited the efforts of ICIS investigators in providing the much-needed data to shift the focus from platelet count to bleeding phenotype and anticipated that future meetings will evaluate the results of collaborative work in the validation and utility bleeding scores as well as the impact of therapeutic choices in the quality-of-life for patients and families.

Open Access This article is distributed under the terms of the Creative Commons Attribution Noncommercial License which permits any noncommercial use, distribution, and reproduction in any medium, provided the original author(s) and source are credited.

\section{References}

1. Bolton-Maggs PH, Moon I (1997) Assessment of UK practice for management of acute childhood idiopathic thrombocytopenic purpura against published guidelines. Lancet 350(9078):620-623

2. George JN, Woolf SH, Raskob GE, Wasser JS, Aledort LM, Ballem PJ et al (1996) Idiopathic thrombocytopenic purpura: a practice guideline developed by explicit methods for the American Society of Hematology. Blood 88(1):3-40

3. Kuhne T, Buchanan GR, Zimmerman S, Michaels LA, Kohan R, Berchtold W et al (2003) A prospective comparative study of 2540 infants and children with newly diagnosed idiopathic thrombocytopenic purpura (ITP) from the Intercontinental Childhood ITP Study Group. J Pediatr 143(5):605-608

4. Lilleyman JS (1994) Intracranial haemorrhage in idiopathic thrombocytopenic purpura. Paediatric Haematology Forum of the British Society for Haematology. Arch Dis Child 71(3):251-253

5. Neunert CE, Buchanan GR, Imbach P, Bolton-Maggs PH, Bennett CM, Neufeld EJ et al (2008) Severe hemorrhage in children with newly diagnosed immune thrombocytopenic purpura. Blood 112 (10):4003-4008

6. Buchanan GR, Adix L (2002) Grading of hemorrhage in children with idiopathic thrombocytopenic purpura. J Pediatr 141(5):683-688
7. Page LK, Psaila B, Provan D, Michael Hamilton J, Jenkins JM, Elish AS et al (2007) The immune thrombocytopenic purpura (ITP) bleeding score: assessment of bleeding in patients with ITP. Br J Haematol 138(2):245-248

8. Jayabose S, Levendoglu-Tugal O, Ozkaynkak MF, Visintainer P, Sandoval C (2004) Long-term outcome of chronic idiopathic thrombocytopenic purpura in children. J Pediatr Hematol Oncol 26(11):724-726

9. Tarantino MD (2000) Treatment options for chronic immune (idiopathic) thrombocytopenia purpura in children. Semin Hematol 37(1 Suppl 1):35-41

10. Neunert CE, Bright BC, Buchanan GR (2008) Severe chronic refractory immune thrombocytopenic purpura during childhood: a survey of physician management. Pediatr Blood Cancer 51 (4):513-516

11. Emilia G, Luppi M, Morselli M, Forghieri F, Potenza L, Torelli G (2008) A possible role for low-dose cyclosporine in refractory immune thrombocytopenic purpura. Haematologica 93(7):1113-1115

12. Hou M, Peng J, Shi Y, Zhang C, Qin P, Zhao C et al (2003) Mycophenolate mofetil (MMF) for the treatment of steroidresistant idiopathic thrombocytopenic purpura. Eur J Haematol 70(6):353-357

13. Sobota A, Neufeld EJ, Lapsia S, Bennett CM (2009) Response to mercaptopurine for refractory autoimmune cytopenias in children. Pediatr Blood Cancer 52(1):80-84

14. Vesely SK, Perdue JJ, Rizvi MA, Terrell DR, George JN (2004) Management of adult patients with persistent idiopathic thrombocytopenic purpura following splenectomy: a systematic review. Ann Intern Med 140(2):112-120

15. Crary SE, Buchanan GR (2009) Vascular complications after splenectomy for hematologic disorders. Blood 114(14):28612868

16. Arnold DM, Dentali F, Crowther MA, Meyer RM, Cook RJ, Sigouin C et al (2007) Systematic review: efficacy and safety of rituximab for adults with idiopathic thrombocytopenic purpura. Ann Intern Med 146(1):25-33

17. Hasan A, Michel M, Patel V, Stasi R, Cunningham-Rundles S, Leonard JP et al (2009) Repeated courses of rituximab in chronic ITP: three different regimens. Am J Hematol 84(10):661-665

18. Parodi E, Rivetti E, Amendola G, Bisogno G, Calabrese R, Farruggia $\mathrm{P}$ et al (2009) Long-term follow-up analysis after rituximab therapy in children with refractory symptomatic ITP: identification of factors predictive of a sustained response. Br J Haematol 144(4):552-558

19. Mueller BU, Bennett CM, Feldman HA, Bussel JB, Abshire TC, Moore TB et al (2009) One year follow-up of children and adolescents with chronic immune thrombocytopenic purpura (ITP) treated with rituximab. Pediatr Blood Cancer 52(2):259-262

20. Zaja F, Battista ML, Pirrotta MT, Palmieri S, Montagna M, Vianelli $\mathrm{N}$ et al (2008) Lower dose rituximab is active in adults patients with idiopathic thrombocytopenic purpura. Haematologica 93(6):930-933

21. Godeau B, Porcher R, Fain O, Lefrere F, Fenaux P, Cheze S et al (2008) Rituximab efficacy and safety in adult splenectomy candidates with chronic immune thrombocytopenic purpura: results of a prospective multicenter phase 2 study. Blood 112(4):999-1004

22. Aksoy S, Harputluoglu H, Kilickap S, Dede DS, Dizdar O, Altundag K et al (2007) Rituximab-related viral infections in lymphoma patients. Leuk Lymphoma 48(7):1307-1312

23. Carson KR, Evens AM, Richey EA, Habermann TM, Focosi D, Seymour JF et al (2009) Progressive multifocal leukoencephalopathy after rituximab therapy in HIV-negative patients: a report of 57 cases from the Research on Adverse Drug Events and Reports project. Blood 113(20):4834-4840

24. Di Carlo I, Primo S, Pulvirenti E, Toro A (2008) Should all splenectomised patients be vaccinated to avoid OPSI? Revisiting 
an old concept: an Italian retrospective monocentric study. Hepatogastroenterology 55(82-83):308-310

25. Okabayashi T, Hanazaki K (2008) Overwhelming postsplenectomy infection syndrome in adults - a clinically preventable disease. World J Gastroenterol 14(2):176-179

26. Kuhne T, Blanchette V, Buchanan GR, Ramenghi U, Donato H, Tamminga RY et al (2007) Splenectomy in children with idiopathic thrombocytopenic purpura: a prospective study of 134 children from the Intercontinental Childhood ITP Study Group. Pediatr Blood Cancer 49(6):829-834

27. Nurden AT, Viallard JF, Nurden P (2009) New-generation drugs that stimulate platelet production in chronic immune thrombocytopenic purpura. Lancet 373(9674):1562-1569

28. Zhou Z, Yang L, Chen Z, Chen X, Guo Y, Wang X et al (2007) Health-related quality of life measured by the Short Form 36 in immune thrombocytopenic purpura: a cross-sectional survey in China. Eur J Haematol 78(6):518-523

29. McMillan R, Bussel JB, George JN, Lalla D, Nichol JL (2008) Selfreported health-related quality of life in adults with chronic immune thrombocytopenic purpura. Am J Hematol 83(2):150-154

30. Klaassen RJ, Blanchette VS, Barnard D, Wakefield CD, Curtis C, Bradley CS et al (2007) Validity, reliability, and responsiveness of a new measure of health-related quality of life in children with immune thrombocytopenic purpura: the Kids' ITP Tools. J Pediatr 150(5):510-515, 5 e1

31. Neunert CE, Buchanan GR, Blanchette V, Barnard D, Young NL, Curtis C et al (2009) Relationships among bleeding severity, health-related quality of life, and platelet count in children with immune thrombocytopenic purpura. Pediatr Blood Cancer 53 (4):652-654 